\title{
Sustainable Design Approach: A case study of BIM use
}

\author{
Wael Abdelhameed \\ Department of Architecture and Interior Design, University of Bahrain \\ wabdelhameed@uob.edu.bh; wael.abdelhameed@gmail.com
}

\begin{abstract}
Achieving sustainable design in areas such as energy-efficient design depends largely on the accuracy of the analysis performed after the design is completed with all its components and material details. There are different analysis approaches and methods that predict relevant values and metrics such as U value, energy use and energy savings. Although certain differences in the accuracy of these approaches and methods have been recorded, this research paper does not focus on such matter, where determining the reason for discrepancies between those approaches and methods is difficult, because all error sources act simultaneously. The research paper rather introduces an approach through which BIM, building information modelling, can be utilised during the initial phases of the designing process, by analysing the values and metrics of sustainable design before going into the design details of a building.

Managing all of the project drawings in a single file, BIM -building information modelling- is well known as one digital platform that offers a multidisciplinary detailed design -AEC model (Barison and Santos, 2010, Welle et.al., 2011). The paper presents in general BIM use in the early phases of the design process, in order to achieve certain required areas of sustainable design. The paper proceeds to introduce BIM use in specific areas such as site selection, wind velocity and building orientation, in terms of reaching the farther possible sustainable solution.

In the initial phases of designing, material details and building components are not fully specified or selected yet. The designer usually focuses on zoning, topology, circulations, and other design requirements. The proposed approach employs the strategies and analysis of BIM use during those initial design phases in order to have the analysis and results of each solution or alternative design. The stakeholders and designers would have a better effective decision making process with a full clarity of each alternative's consequences. The architect would settle down and proceed in the alternative design of the best sustainable analysis. In later design stages, using the sustainable types of materials such as insulation, cladding, etc., and applying sustainable building components such as doors, windows, etc. would add more improvements and enhancements in reaching better values and metrics.
\end{abstract}

The paper describes the methodology of this design approach through BIM strategies adopted in design creation. Case studies of architectural designs are used to highlight the details and benefits of this proposed approach. 


\section{Sustainable Architectural Design}

According to Wikipedia definition, the basic objectives of sustainability are to reduce consumption of non-renewable resources, minimize waste, and create healthy, productive environments.

Sustainability in architectural design seeks lower energy and water consumption; limit resource consumption through an emphasis on water recycling and solar energy; give priorities to non-toxic materials; minimize impact on climate change by reducing gas emissions; and emphasize quality and durability over price.

Sustainable architectural design is to design the built environment to respect the principles of social, economic and ecological sustainability. It attempts at reducing negative impacts on the environment in general, and the comfort of building occupants in particular. Its main objective, therefore, is to improve building performance in terms of the three main areas: society, economy and ecology.

Although the three main areas of sustainability are society, economy and ecology, this research paper is exclusively concerned with the ecological factors, the environmental factors and their impact on building performance at the initial design phases before the design is finalised. The first area, social factors, is viewed as immaterial values that would distinctly vary from one society to another. It therefore would preferably be evaluated by the architects based on the design location and the society values and customs. The second area, economic factors, has more obvious effect and impact in the final architectural design phases in selecting the finishing materials, more than in the initial phases of the design process itself.

In summary, the scope of this research paper is the environmental factors on building performance during the initial design phases. The paper focuses on small size projects where architects in the most cases are the only designers.

\section{The Current Architectural Education and Practice}

There are many factors and requirements that are taken into account during the architectural design process. Architectural design solution should satisfy the whole set of those interrelated factors and requirements. It is often necessary to develop an integrated solution for a whole set of interrelated requirements (Lawson, 2005).

The architect during conceptual designing process attempts to respect main factors namely: aesthetics, functions and economics. The architect seeks composing and 
proposing forms and spaces through required relationships and certain topologies in order to have a satisfactory design solution (Abdelhameed, 2004).

Other factors may influence the architectural design are: climate -weather temperature, sunshine and sun path, relative humidity, wind direction and speed-; topography -contour lines, nearby water surfaces, plateaus and mountains-; site limitations and considerations -main and service entrances, infrastructure connections, outdoor spaces-; urban design -surrounding urban fabric, road network, surrounding building materials and structural systems-; structural and technical issues -structural systems, building materials, mechanical and electrical requirements-.

The architecture curriculums in the theoretical level, as well as architectural design offices in the professional level, focus during the initial design phases and conceptual designing on these foregoing factors. In the practical and professional level, the environmental factors analysis used to be conducted at the final stages or after the design is completed, specifically in cases of small and medium size projects in which the designer is a small team of architects only with no other discipline specialists.

\section{The Proposed Method}

Architects should apply the analysis of different disciplines in order to achieve optimal design solutions in terms of electrical, mechanical, solar, thermal, energy, acoustic analysis. The early analysis in design stages helps reduce costs and increase occupant satisfaction.

The proposed method is to include the environmental factors analysis among the requirements of the design process, consequentially in the theoretical level the design evaluation inside the design studios of architecture schools should highlight this importance, and in the practical and professional level the different kinds of environmental analysis have to be performed to achieve the optimal solution before betaking to and relying on different assistances such as expensive insulation materials.

The proposed method employs BIM, building information modelling, as a single platform of the design to perform the environmental factors analysis, by using Autodesk programs, Revit as a BIM program and Green Building Studio as energy analysis and simulation program, versions 2014 and 2015. Having one platform would help architects (Kim et.al., 2015, Schlueter and Thesseling, 2009) and 
architecture students (Sebastian and van Berlo, 2010) effectively conduct the design and the environmental analysis without the assistance of other specialists or the knowledge of other energy analysis programs such as eQuest.

The research method focuses on the change that may need to be made in the design before starting in the working drawings and design details. Therefore, the researcher asked the students to model their previous conceptual designs using BIM, Revit program, and to conduct the environmental analysis on their designs. The objective is to discover whether these designs can be slightly modified and changed to improve the design performance or not.

The main reason of applying only slight change or modification is to keep the design satisfying other architectural design requirements that were discussed in the previous part of the research paper. This condition was highlighted to the students. Any change should not contradict with any other architectural requirement such as, function, structural, technical, etc.

\section{Research Methodology}

The research paper attempts at applying and investigating the proposed method in different areas of sustainability in design cases, by measuring environmental factors such as: solar analysis, thermal analysis, shadows and reflections, ventilation and air flow.

The research paper employs qualitative analysis of architectural design case studies to investigate the effectiveness of the proposed method. The researcher's students applied the environmental analysis in different design case studies. Each student was asked to apply one area of environmental analysis on a design, and to record the change in the design that may improve the design performance.

The research paper presents the qualitative analysis of each design case, and what were the benefits, if any, that were added to the design.

\section{Architectural Design Cases and Qualitative Analysis}

The researcher recorded the steps used in each design case according to the emphases area of environmental analysis. Any change in the architectural design was discussed with the students in order to ensure the consistency of modification level.

There were some architectural considerations, in few design cases, which are not used in this research paper because the change was architecturally significant. However, the proposed method was effective even in those cases. Clear 
improvements were recorded after applying the environmental analysis in those design cases.

No comparison between design case studies was used or conducted due to having a different environmental area and application in each design case. Nevertheless, the improvement in each design case was evident.

\section{Site Selection}

The environmental analysis in this case study was conducted to compare between different proposed sites that satisfy the project requirements. Figure 1 displays the results of the best site in terms of electricity consumption while Figure 2 displays the environmental analysis of different sites.

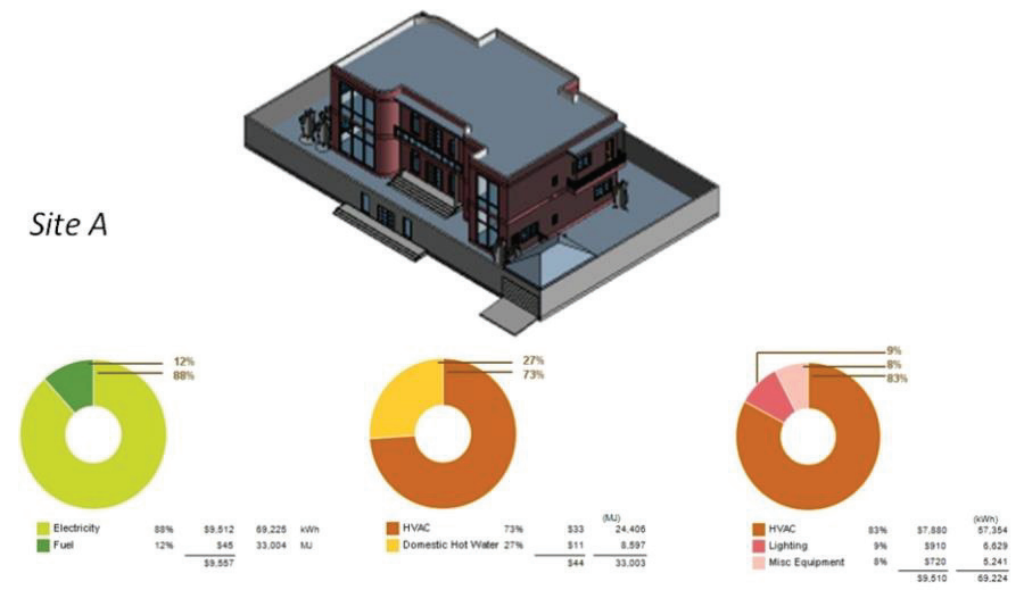

Figure 1 Environmental analysis of the selected site 


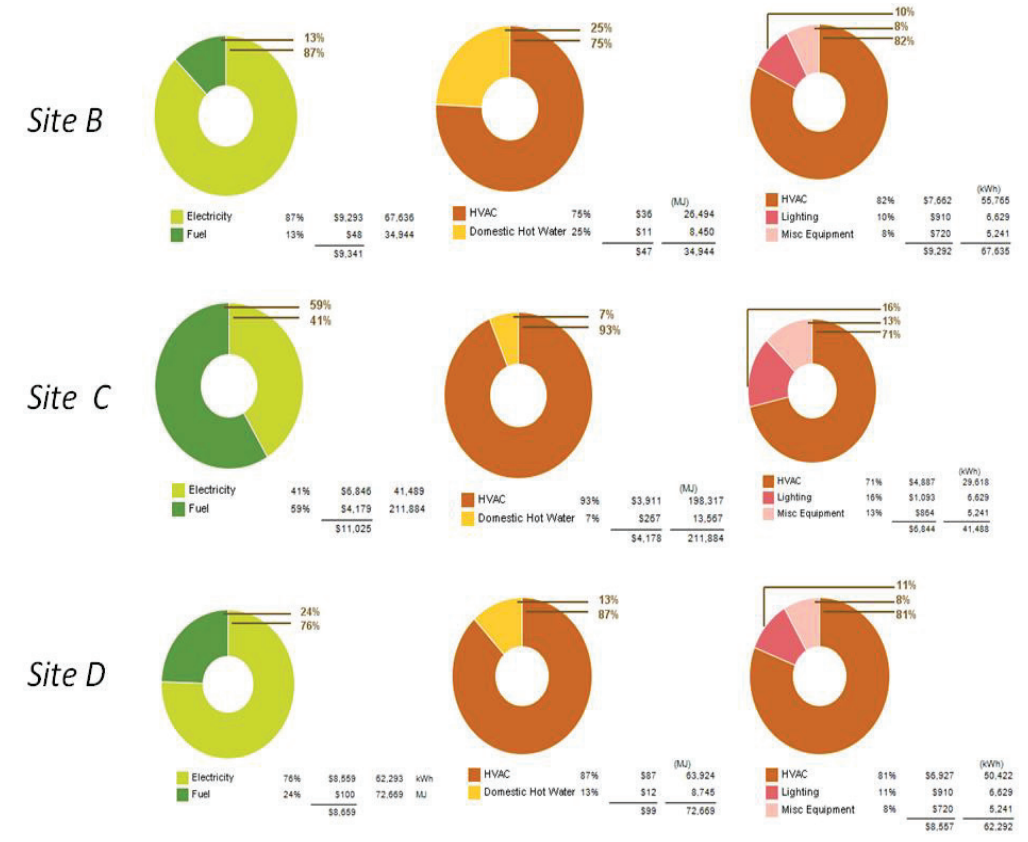

Figure 2 Environmental analysis of the proposed sites of a design project

\section{Orientation Analysis}

In this design case study, the question was how the angle between building masses would improve the building performance. The student conducted environmental analysis of some angles -30, 45, 60, 80 and 90 degrees- that would not apply any change in the conceptual design. According the analysis, the best angle between the building masses was 30 degrees to achieve the required wind speed, Figure 3. 

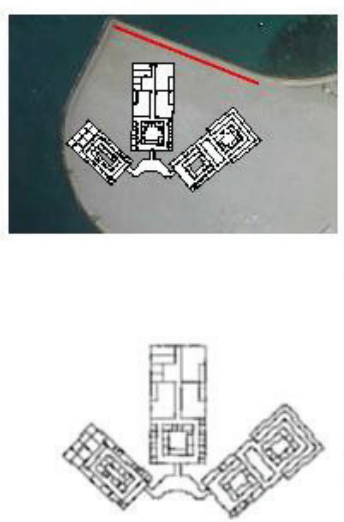

30 Degree Angle between the wings of the building
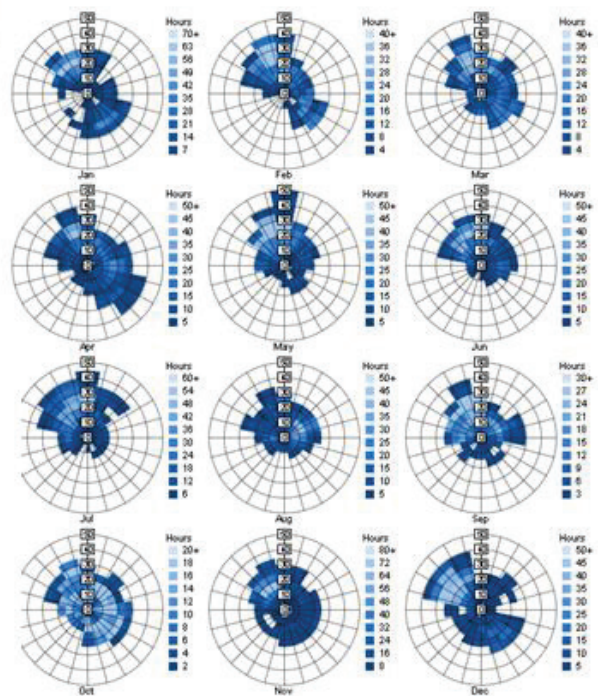

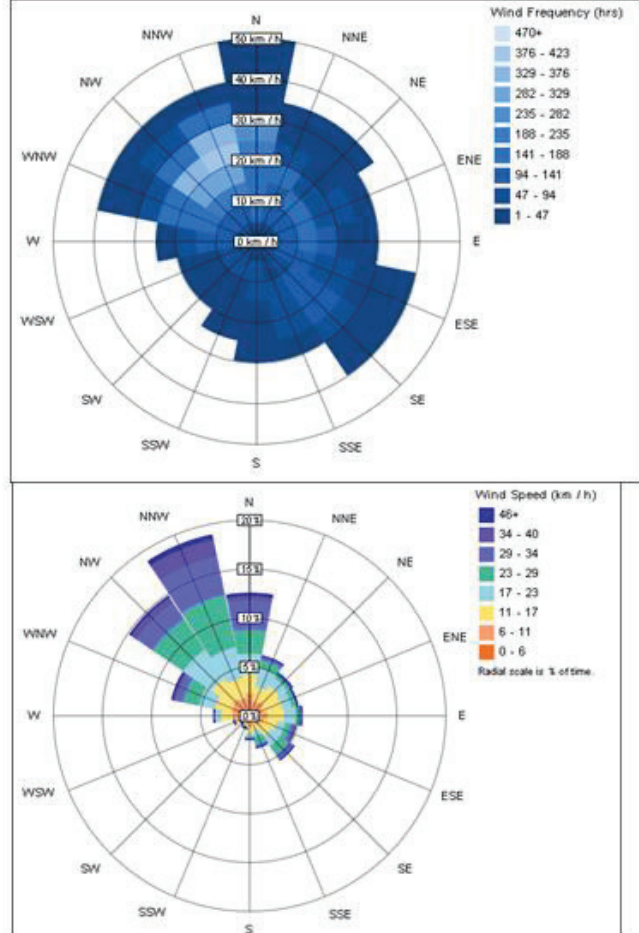

Figure 3, An example of BIM analysis to decide the best angle between the building masses

Another case study of orientation analysis was to specify the best orientation of a courtyard inside the design to achieve the optimal cooling solution, Figure 4 and 5.

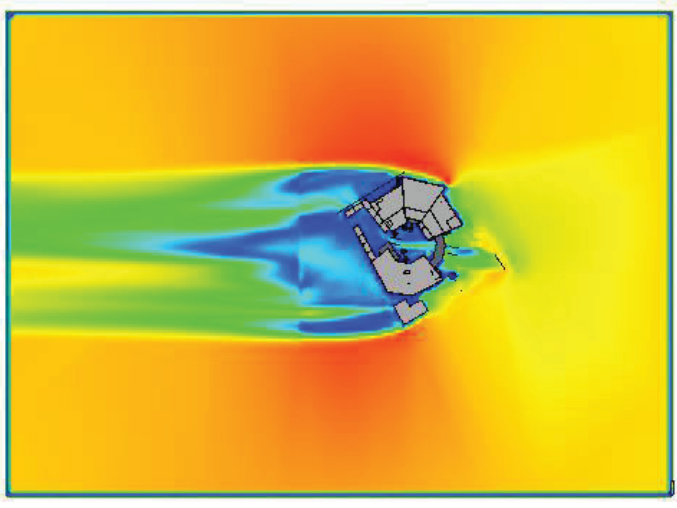

Figure 4, Simulation of environmental analysis in the plan view 


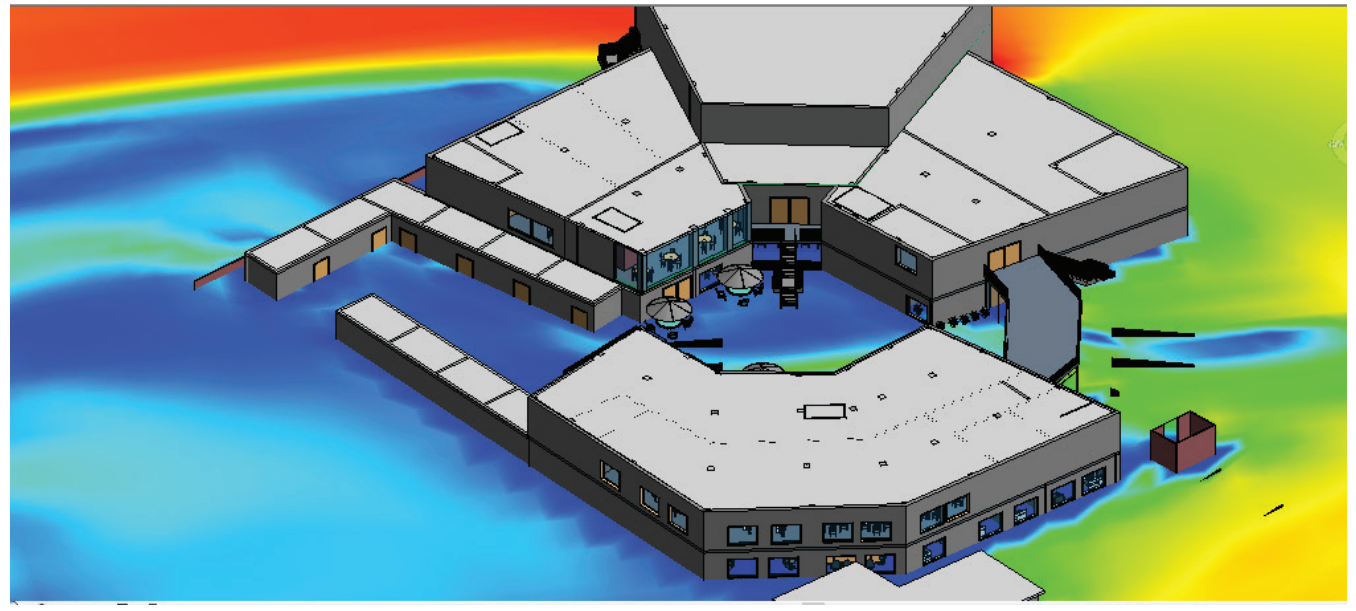

Figure 5, Simulation of environmental analysis to show the best cooling result

\subsection{Energy Saving Analysis}

The environmental analysis in the area of energy saving was conducted to get data of thermal, temperature and wind. Data analysis simulation on the building envelope helped the student decide simple changes in the architectural design, such as projecting the first floor in order to shade the main entrance, Figure 6 and 7.

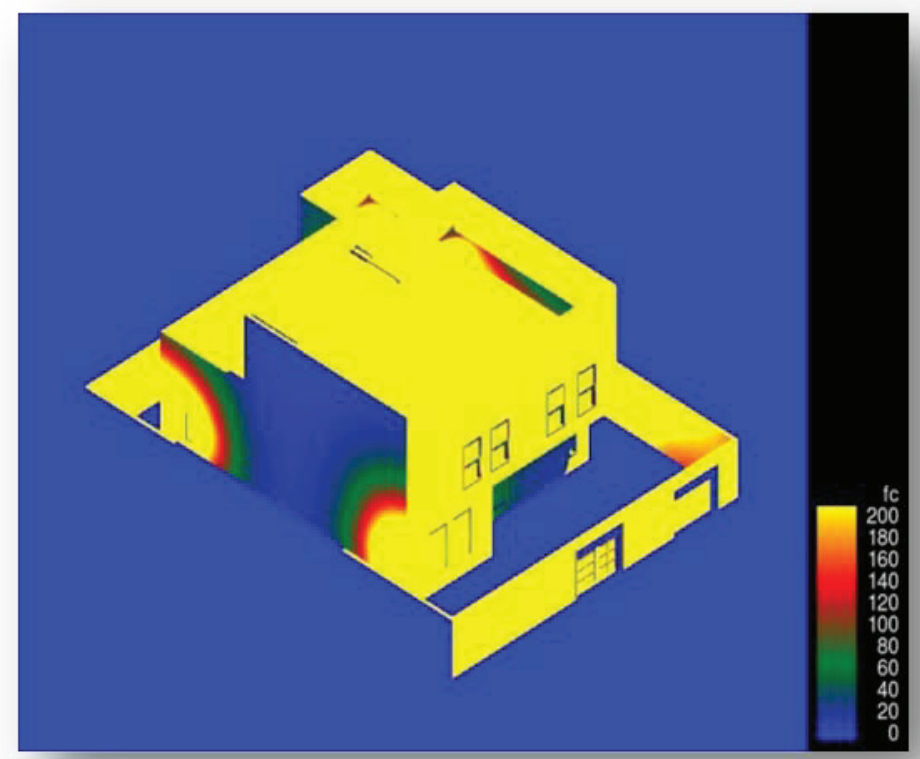

Figure 6, Data simulation on the building envelope. 

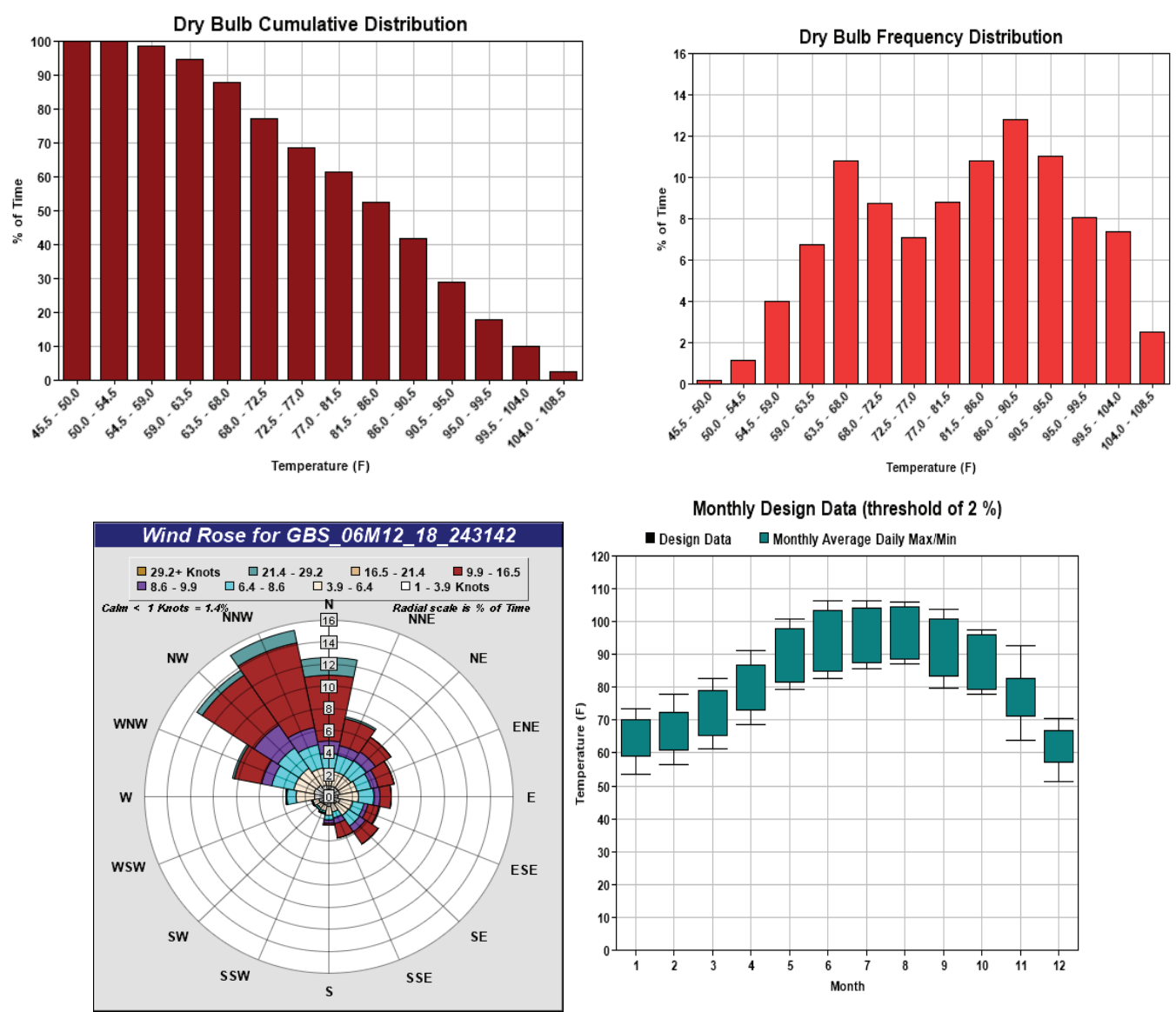

Figure 7, Energy data results of the modified solution

\section{U-Value Calculations}

Since the U-value is a measurement of heat flow, the lower the U-value, the more slowly does the material transfer heat in and out of a building. As per Bahrain constriction law, the overall thermal transmittance value -U-value- for the walls should not be more than $0.75 \mathrm{~W} / \mathrm{m}^{2} .{ }^{\circ} \mathrm{C}$ (Mohanadi, 2006).

In this case study, a plug-in for U value calculations was used inside the Revit program to help the student save time and effort. The plug-in automatically calculates U-Value -Overall heat transfer coefficient- for walls, floors, roofs, ceilings and foundation slabs.

Based on the result, the student used a double layer wall with an air cavity in the middle which reduced the U-Value to the target value $0.75 \mathrm{~W} / \mathrm{m}^{2} .{ }^{\circ} \mathrm{C}$, Figure 8 . 

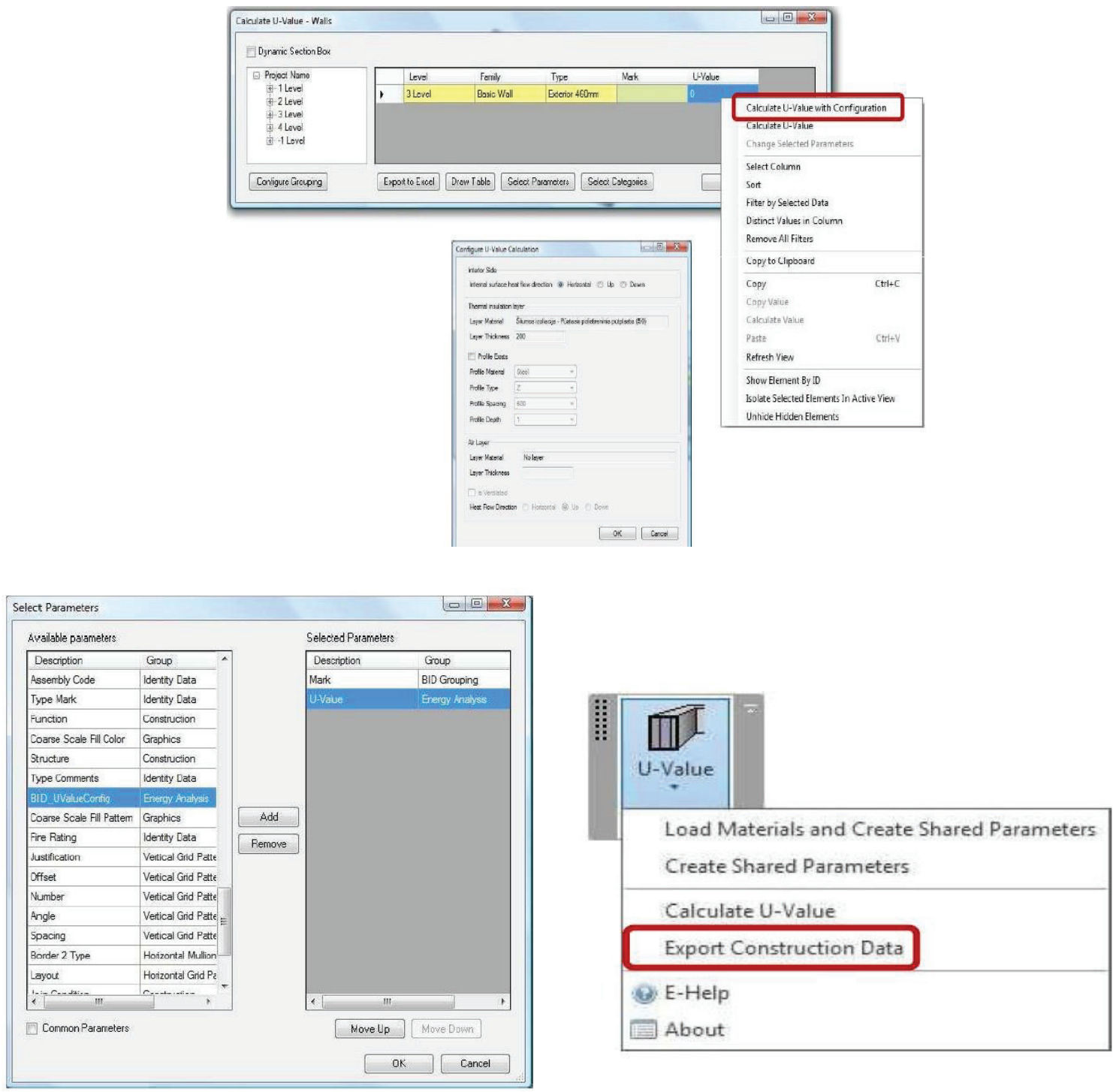

Figure 8, 3 screen shots of the Plug-In for U-Value calculations in the student project

In all design cases, every student presented a slight change in the conceptual design to clearly improve the existing conceptual design. This evidence not only proves the effectiveness of the proposed method but also highlights that the lack of awareness and the importance of environmental analysis.

\section{Conclusion and Recommendations}

The analysis recorded by the research paper proved the effectiveness of the proposed method. In all architectural design cases, the building performance was improved by 
applying a slight architectural change that does not affect the main project requirements and objectives.

The different environmental analysis should be conducted to each design even at small size projects. More awareness should be achieved and spread among architects and stakeholders to highlight the importance of environmental analysis in the conceptual design phases.

Architecture curriculums should concentrate on the environmental analysis in design studios with the same value and scale as other design requirements such as structure, site, urban design, etc.

\section{References}

1. Kim, J.B., Jeong, W., Clayton, M.J., Haberl, J.S. and Yan, W.: Developing a physical BIM library for building thermal energy simulation, Automation in construction, 50, 16-28 (2015).

2. Lawson, B.: How designers think: the design process demystified, Elsevier/Architectural, (2005).

3. Schlueter, A. and Thesseling, F.: Building information model based energy/exergy performance assessment in early design stages, Automation in construction, 18(2), 153-163 (2009).

4. Sebastian, R. and van Berlo, L.: Tool for benchmarking BIM performance of design, engineering and construction firms in the Netherlands. Architectural Engineering and Design Management, 6(4), 254-263 (2010).

5. Welle, B., Haymaker, J. and Rogers, Z.: December. ThermalOpt: A methodology for automated BIM-based multidisciplinary thermal simulation for use in optimization environments, Building Simulation (4) 4, 293-313 (2011).

6. Mohanadi, K. k. A.: Thermal insulation in buildings (1st ed.). Kingdom of Bahrain: Ministry of electricity \& water (2006).

7. Abdelhameed, W.: Visual design thinking in the design process as impacted by digital media. Architecture in the Network Society, the 22nd eCAADe Conference Proceedings, 90-94 (2004).

8. Barison, M.B. and Santos, E.T.: BIM teaching strategies: an overview of the current approaches, ICCCBE International Conference on Computing in Civil and Building Engineering (2010). 